\title{
CH50 Activity and its Relationship to Antimüllerian Hormone in Patients with Infertility
}

Hilda Sánchez Hernández, ${ }^{1}$ Héctor Salvador Godoy Morales, ${ }^{1}$ Daniel Vieyra Cortés, ${ }^{1}$ Francisco Miguel Rojas Camacho, ${ }^{1}$ Berenice Flores Maldonado, ${ }^{1}$ Gonzalo de Jesús Siu Moguel, ${ }^{1}$ Victor Saul Vital Reyes, ${ }^{2}$ José Andrés Avilés Martí, ${ }^{2}$ José Manuel Lozano Sánchez ${ }^{2 *}$

${ }^{1}$ Hospital Ángeles Pedregal, Department of Reproductive Medicine, Mexico

${ }^{2}$ Unidad de Reproducción Ciudad de México, México

\begin{abstract}
Objective: To study the importance of immunological screening (CH50, $\mathrm{C} 3, \mathrm{C} 4)$ to determine the correlation between the ovarian reserve and complement pathway.

Design: A cross-sectional multivariate study.

Setting: Single tertiary referral center.

Patients: 40 Women with infertility diagnosis who underwent In Vitro Fertilization (IVF).

Intervention(s): Basal blood samples collected to measure complement pathway and antimüllerian hormone.

Main Outcome Measure(s): To correlate complement pathways (CH50 levels) with AMH.

Results: The complement system and ovarian reserve were studied in 40 women aged from 28-48 years (mean=37.87 years), finding the values of CH50 65-268 CAE (mean=153.2 CAE), C3 13.9- 183mg/dl (mean=128.52mg/dl), C4 11.5-106mg/dl (mean=29.86mg/dl), AMH 0.10-3.9ng/dl (mean $=1.32 \mathrm{ng} / \mathrm{dl}$ ). A multivariate analysis comparison was also conducted yielding significant results: negative correlation between AMH and age (Spearman correlation -0.6; $\mathrm{P}=0.001$ ), as well as between AMH and CH50 (Spearman correlation -0.4; $\mathrm{P}=0.0129$ ).

Conclusion: High CH50 levels are correlated with an AMH decrease and it could be accordingly associated with age-related chronic inflammation.

Capsule: High levels of CH50 are correlated with a decrease in AMH levels and could also be considered as a marker of poor response in IVF cycles.
\end{abstract}

Keywords: CH50, AMH, Immune infertility, Complement pathways, ovarian reserve

\section{Introduction}

In modern age, behavior and lifestyle have changed dramatically due to modifications in fertility factors. Advanced maternal age, inflammatory alterations, and concomitant diseases can change fertility rate over time. We can use the ovarian reserve as a fertility predictor since it is a concept that reflects the quality and quantity of oocytes present in the ovary. ${ }^{1,2}$ In addition to measuring AMH to quantify the ovarian reserve other basal serum markers can be taken into account: inhibition B, follicle-stimulating hormone (FSH), and estradiol, as well as ultrasonographic markers such as ovarian volume measurement or antral follicle count (AFC). ${ }^{1} \mathrm{AMH}$ is a dimeric glycoprotein, discovered in the 1940s, that participates in sexual differentiation, and was found in human follicles in 1990. It is produced by developing follicle granulosa cells and with clear clinical significance in ovarian function and reserve. ${ }^{2}$ Clinical applications of AMH serum measurement include menopause prediction in different patient groups, for example, it can be predicted in the general population, or in patients with a history of ovarian surgery who have received chemotherapy or radiation therapy, who have

\begin{tabular}{|l|l|}
\hline \hline Quick Response Code: & *Corresponding author: José Manuel Lozano Sánchez, Unidad de Reproducción Ciudad de \\
México, México. Av. Río Mixcoac 274, Acacias, Benito Juárez, 03240 Ciudad de México, México \\
Received: 03 August, 2021 & Published: 30 August, 2021 \\
Citation: Hernández HS, Morales HSG, Cortés DV, Camacho FMR, Maldonado BF, et al. CH50 \\
Activity and its Relationship to Antimüllerian Hormone in Patients with Infertility. Curr Inv Cln \\
Med Res. 2021;1(3):1-5. DOI: 10.53902/CICMR.2021.01.000513
\end{tabular}


had immunological and/or inflammatory diseases, endometriosis, or polycystic ovary syndrome (PCOS), among others. ${ }^{2-4}$

The main factor that causes a negative impact on AMH levels is age, as it decreases over time, as well as the number of total oocytes decreases. Currently, it is known that on average levels it falls $0.384 \mathrm{ug} / \mathrm{L}$ per year. ${ }^{4}$

Premature ovarian insufficiency (POI) is a reproductive endocrine disorder, caused by follicular dysfunction in women under 40 years of age who have increased levels of FSH (>40IU/L), usually diagnosed in patients with Turner syndrome, ovarian autoimmunity, infections, chemotherapy/radiation therapy, and in some cases without an understandable cause. In such conditions, AMH levels may be undetectable. ${ }^{4}$ Regarding autoimmune POI, there is a theory called "autoimmune oophoritis", which acknowledges that there is a selective alteration of developing follicles causing a progressive decrease in ovarian function and reserve, featured by inflammatory cell infiltrate (plasma cells, B cells, and T cells) in theca cells of growing follicles. It is said to be selective since follicles in early stages have not been detected. ${ }^{5}$ However, it is not known whether the association between low ovarian reserve could be due to chronic treatments with medications such as methotrexate, or secondary to an inflammatory process itself. ${ }^{6}$

Inflammatory pelvic disease (IPD) has shown a direct detrimental effect on the pelvic environment and endometrial receptivity. Despite this fact, the main mechanism in which chronic IPD can destroy the ovarian reserve is not clear considering quantitative and qualitative follicle damage. Patients with Crohn's disease or dermatomyositis have decreased $\mathrm{AMH}$ and $\mathrm{AF}$, suggesting that inflammation causes a direct negative effect on follicles. ${ }^{7}$ Endometriosis can cause an increase in the number of ovarian cytokines, which can cause ovarian damage and a decrease in AMH levels. ${ }^{8}$ AMH in women with endometriosis is detected at lower levels compared to the general population; endometrioma management can by itself cause a decrease in follicle count. ${ }^{9}$ Other factors associated with inflammation that may modify AMH concentrations include overweight, ethnicity, vitamin D deficiencies, AMH polymorphisms, and its receptors and genome variations. ${ }^{10}$

The complement system is key to the study of the innate immune system, its activation involves a cascade of proteases that provide the first line of defense against pathogens and plays an important role in the elimination of cellular detritus. It reinforces the function of phagocytes and antibodies by eliminating microorganisms, removing apoptotic cells and cell detritus, increasing levels of inflammation (by chemo-attracting leukocytes, macrophages, and neutrophils), and activating costimulation of B cells, thus increasing the innate and adaptive response. ${ }^{11}$ The study of CH50, C3, and $\mathrm{C} 4$ is a measure to be taken to evaluate the inflammatory response of the complement since they are sensitive to the reduction, absence, or inactivity of any of the complement pathways. ${ }^{12}$ Acute and chronic inflammation allows an increase in the proteins of the complement, which are acute-phase reactants and synthesized in the liver responding to inflammatory cytokines. ${ }^{8}$

\section{Material and Methods}

A cross-sectional study was conducted on a pre-existing prospective cohort of patients who come to the fertility clinic diagnosed with infertility, who had been indicated with high complexity treatment (IVF) from January 2019 to May 2020.

\section{Sample selection}

46 patients diagnosed with infertility (primary or secondary) who underwent high complexity therapy (IVF) were evaluated from January 2019 to May 2020. In order to evaluate the ovarian reserve, complement levels, and immune profile, blood samples were taken on day 3 of the menstrual cycle, where they are included for the complement study: CH50 (CAE), C3 (mg/dl), C4 (mg/dl); for ovarian reserve AMH (ng/d), FSH (mIU/ml), $\mathrm{LH}(\mathrm{mIU} / \mathrm{ml})$, and estradiol (ng/dl) levels were measured. Thyroid function was also evaluated with a thyroid-stimulating hormone (TSH) test and an immunological profile was taken, including antinuclear antibodies (ANA), rheumatoid factor (RF) (IU/ml), double-stranded DNA antibodies (IU/ml), C-reactive protein (CRP) $(\mathrm{mg} / \mathrm{L})$, and erythrocyte sedimentation rate $(\mathrm{ESR})(\mathrm{mm} / \mathrm{h})$.

\section{Inclusion criteria}

The study includes all patients diagnosed with infertility who have undergone IVF treatment from January 2019 to May 2020, and a complete assessment of the complement pathways and ovarian reserve studies, as well as vaginal cultures: exudate, mycoplasma, negative ureaplasma, and chlamydia.

\section{Exclusion criteria}

Patients with incomplete data, who do not wish to carry out the study, as well as patients with a history of pre-study diagnosed immunological disease, patients diagnosed with chronic diseases (systemic high blood pressure, diabetes mellitus, hiccups, or hyperthyroidism) are excluded.

\section{Elimination criteria}

Patients who refer to chronic use of any medicine.

\section{Statistical analysis}

A multivariate analysis was performed with JMP® version 12 . For comparative analysis, a correlation of Spearman with a significant $\mathrm{P}<0.05$ was used.

\section{Results}

46 patients diagnosed with infertility and IVF candidates aged between 28 and 48 years (average age: 37.87 years) were evaluat- 
ed. 6 patients are excluded from the study, 2 diagnosed with chronic high blood pressure, and 4 with hypothyroidism without adequate treatment. Finally, 40 patients were included, of whom 17 patients were diagnosed with primary infertility and 23 with secondary infertility.

Basal hormonal profile (on day 3 of the menstrual cycle) where ovarian reserve markers were evaluated presenting the following results: $\mathrm{AMH}$ in a range of 0.1 to $3.9 \mathrm{ng} / \mathrm{dl}(1.32 \mathrm{ng} / \mathrm{dl}), \mathrm{FSH} 1.40-$ $71.31 \mathrm{mIU} / \mathrm{ml}(10.00 \mathrm{mIU} / \mathrm{ml})$, LH $1.26-41.16 \mathrm{mIU} / \mathrm{ml}(8.42 \mathrm{mIU} /$ $\mathrm{ml}$ ) and estradiol $10-1205 \mathrm{pg} / \mathrm{dl}$ (150pg/dl); in the same sample complement pathways were evaluated finding CH50 65-268 CAE (153.2 CAE), total C3 $13.9-183 \mathrm{mg} / \mathrm{dl}(128.52 \mathrm{mg} / \mathrm{dl})$, total C4 $11.5-106 \mathrm{mg} / \mathrm{dl}(29.86 \mathrm{mg} / \mathrm{dl}) ;$ TSH $0.58-5.16$ (2.16), ANA of 0.10 1.98 (0.58), rheumatoid factor $6-66.80 \mathrm{IU} / \mathrm{ml}(11.12 \mathrm{IU} / \mathrm{ml})$, double-stranded DNA antibodies 2.6-200IU/ml (20.36 IU/ml), ESR 9.37 (2-22) mm/h, and CRP 0.49 (0.1-1.2) mg/L (Table 1).

During the study, 2 patients were diagnosed with subclinical hypothyroidism, 1 with hyperthyroidism, and 2 were diagnosed with systemic lupus erythematosus. In the statistical analysis, a significant correlation was found between age and AMH assessed with a Spearman's rank correlation of 0.6 and $\mathrm{P}=0.001$; as well as a significant correlation between the CH50 and AMH values, finding a Spearman correlation of -0.4 and $\mathrm{P}=0.0129$ (Figure 1); the remain- ing main variables compared did not show any significant results (Table 2).

Table 1: Demographic and clinical characteristics of study participants.

\begin{tabular}{|c|c|}
\hline & $\mathrm{n}=40$ \\
\hline Age & $37(28-48)$ years \\
\hline Infertility Type & 17 \\
\hline Primary & 23 \\
\hline Secondary & $153(65-286) \mathrm{CAE}$ \\
\hline CH50 (CAE 63-145) & $128(13-183) \mathrm{mg} / \mathrm{dl}$ \\
\hline C3 (mg/dl 83-193) & $29(11-106) \mathrm{mg} / \mathrm{dl}$ \\
\hline C4 (mg/dl 15-57) & $1.32(0.10-3.9) \mathrm{ng} / \mathrm{dl}$ \\
\hline AMH & $2.16(0.58-5.16)$ \\
\hline TSH & $0.58(0.1-1.98)$ \\
\hline ANA & $11.12(6-66.80) \mathrm{IU} / \mathrm{ml}$ \\
\hline RF (IU/ml) & $20.36(2.6-200) \mathrm{IU} / \mathrm{ml}$ \\
\hline Anti-double stranded DNA & $9.37(2-22) \mathrm{mm} / \mathrm{h}$ \\
ESR (mm/h) & $0.49(0.1-1.2) \mathrm{mg} / \mathrm{L}$ \\
\hline CRP (mg/L) & \\
\hline
\end{tabular}

Note: AMH: Antimüllerian Hormone; TSH: Thyroid Stimulating Hormone; ANA: Anti Nuclear Antibodies; RF: Rheumatoid Factor; DNA: Deoxyribonucleic Acid; ESR: Erythrocyte Sedimentation Rate Taste; CRP: C-Reactive-Protein

After analyzing the secondary variables of the study, they were of no statistical significance (Table 3).

Table 2: Correlation between age, AMH, and complement.

\begin{tabular}{|c|c|c|c|c|}
\hline Variable & Variables & Spearman & Prob $>|\boldsymbol{\rho}|$ & \\
\hline CH50 (CAE 63-145) & Age & 0.2641 & 0.1042 & +++ \\
\hline C3 (mg/dl 83-193) & Age & 0.0993 & 0.5476 & + \\
\hline C3 (mg/dl 83-193) & CH50 (CAE 63-145) & 0.2458 & 0.1314 & ++ \\
\hline C4 (mg/dl 15-57) & Age & 0.2038 & 0.2132 & ++ \\
\hline C4 (mg/dl 15-57) & CH50 (CAE 63-145) & 0.0035 & 0.9829 & \\
\hline C4 (mg/dl 15-57) & C3 (mg/dl 83-193) & 0.229 & 0.1608 & ++ \\
\hline AMH & Age & -0.5065 & $0.001^{*}$ & --- \\
\hline AMH & CH50 (CAE 63-145) & -0.3946 & $0.0129^{*}$ & --- \\
\hline AMH & C3 (mg/dl 83-193) & -0.2352 & 0.1494 & -- \\
\hline AMH & C4 (mg/dl 15-57) & 0.0938 & 0.5699 & + \\
\hline
\end{tabular}

Note: AMH: Antimüllerian Hormone

*Significant values in the study

Table 3: Spearman's correlation between variables and complement activity (CH50).

\begin{tabular}{|c|c|c|c|c|}
\hline Variable & Variable & Spearman & Prob $>|\rho|$ & \\
\hline C3 (mg/dl 83-193) & \multirow{5}{*}{ CH50 (CAE 63-145) } & 0.2458 & 0.1314 & ++ \\
\hline C4 (mg/dl 15-57) & & 0.0035 & 0.9829 & \\
\hline $\mathrm{RF}(\mathrm{IU} / \mathrm{ml}$ less than 12$)$ & & 0.235 & 0.1498 & ++ \\
\hline ANA (range less than 1.5) & & -0.049 & 0.7671 & \\
\hline Anti-double stranded DNA (IU/ml less than 100$)$ & & $-0.0149 *$ & 0.9284 & \\
\hline
\end{tabular}

Note: ANA: Anti Nuclear Antibodies; RF: Rheumatoid Factor; DNA: Deoxyribonucleic Acid

*Significant values in the study 


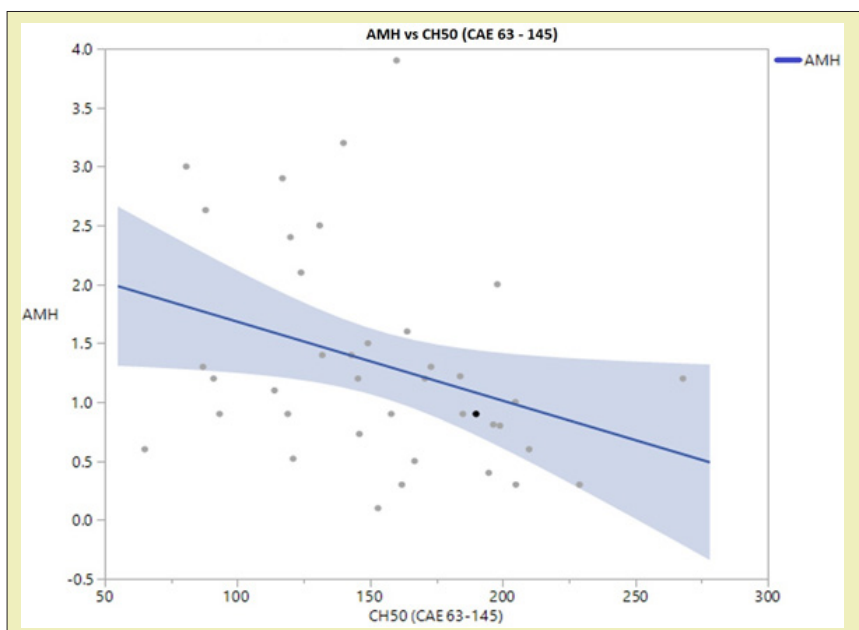

Figure 1: Correlation and simple linear regression analysis displaying that the higher the $\mathrm{CH} 50$ levels, the lower the Antimüllerian hormone (AMH) levels. AMH levels were analyzed in comparison with CH50 to observe Spearman's rank correlation and thus justify those patients showing an increase in CH50 levels (inflammation) closely correspond to a decrease in AMH levels.

\section{Discussion}

The approach to the problem is key to determine the importance of the study since currently, we have found an increasing number of immunological diseases that have an impact on fertility. Nowadays, patients seek answers to problems that were previously inexplicable. Our primary objective was to know the correlation between the complement pathways (CH50, C3, and C4) and AMH levels. By looking at the activity of the complement $\mathrm{CH} 50$, the scope is widely opened for us to know the association between supporting data, such as AMH, which act as a foundation for the study of reproductive age women with infertility. The existing association between decreasing AMH levels and increasing age is well-known. Besides, multiple studies exhibit the association between low AMH levels and low rates of spontaneous pregnancies, as well as unsuccessful responses to IVF cycles. As in this study, Iwase et al, also suggest that AMH is the most used ovarian reserve marker for diagnosis in a wide range of clinical entities that can modify the reserve, however, they do not consider it as a predictor in rates of live newborns. ${ }^{1}$

Bertone-Johnson et al, in an ovarian reserve study, included women aged between 30 and 44 years with fertility problems and evaluated the endocrine profile and ovarian reserve markers (FSH, Inhibin $\mathrm{B}$, estradiol, and $\mathrm{AMH}$ ), concluding that $\mathrm{AMH}$ is a good predictor for age-associated fertility decline. ${ }^{3}$ There are no such studies that show that acute inflammation factors can cause a decrease in AMH levels. For this reason, we also wanted to determine the importance of the complement study, as an alternative in the study of ovarian reserve, associated with inflammation factors in patients with infertility. Some studies refer to low AMH levels and endometriosis, as Kasapoglu et al, who observed that patients with endometriosis have lower basal AMH levels in addition to a faster decrease in them compared to healthy women. ${ }^{13}$ Other researchers associate immunological diseases, like Brouwer et al, who showed an association of rheumatoid arthritis with positive anti-citrullinated antibodies and low AMH levels. However, it is not significant due to the lack of correlation between age and time of treatment with methotrexate. ${ }^{6}$ Cui et al, explained the association between chronic inflammation and the possible mechanisms of the low reserve, thus suggesting special attention in ovarian reserve assessment in patients with a history of pelvic inflammatory disease and infertility. ${ }^{7}$

Up to now, there are no studies available that include inflammation factors as part of the fertility study in patients with a low reserve. Therefore, in this study, we propose the evaluation of complement levels as an ancillary in patients with a proven low ovarian reserve with low AMH levels. Among the study limitations, there was a small sample of patients, whereas, opening a wide spectrum for the study of ovarian reserve in patients with infertility, with which we could consider the complement analysis as an adjuvant for the response of IVF cycles, as well as to rule out any cause of chronic or acute inflammation that we may not be taking into account in the infertility study.

\section{Conclusion}

In this study, we were able to confirm that patients with elevated CH50 levels have lower AMH levels, which in turn could be associated with age-related chronic inflammation. We were also able to consider high CH50 levels as a marker of poor response in IVF cycles. Despite the need for further studies with larger patient cohorts for research in infertility and immune system alterations, this study can open a broader scope regarding the ovarian reserve assessment in patients trying to conceive.

\section{Acknowledgments}

As a team, we would like to honor the opportunity that this journal has given us to share our inquisitive findings as medical researchers in reproductive medicine.

\section{Funding}

None.

\section{Conflicts of Interest}

Authors declare that there are no conflicts of interest.

\section{References}

1. Antonio La Marca, Anna Pia Ferraretti, Roberto Palermo, et al. The use of ovarian reserve markers in IVF clinical practice: a national consensus. Gynecol Endocrinol. 2015;32:1-5.

2. Akira Iwase, Satoko Osuka, Maki Goto, et al. Clinical application of serum anti-Müllerian hormone as an ovarian reserve marker: A review of recent studies. J ObstetGynaecol Res. 2015;44:998-1006.

3. Elizabeth R Bertone Johnson, JoAnn E Manson, Alexandra C Pur- 
due Smithe, et al. Anti-Müllerian hormone levels and incidence of early natural menopause in a prospective study. Hum Reprod. 2018;33:1175-1182.

4. Jure Bedenk, Eda Vrtačnik-Bokal, Irma Virant Klun. The role of antiMüllerian hormone (AMH) in ovarian disease and infertility. J Assist Reprod Genet. 2019;37:89-100.

5. Silva CA, Yamakami LYS, Aikawa NE, et al. E. Autoimmune primary ovarian insufficiency. Autoimmun Rev. 2014;13:427-430.

6. Brouwer J, Dolhain R, Hazes J, et al. Reduced Ovarian Function in Female Rheumatoid Arthritis Patients Trying to Conceive. ACR Open Rheumatology. 2019;1:327-335.

7. Cui L, Sheng Y, Sun M, et al. Chronic Pelvic Inflammation Diminished Ovarian Reserve as Indicated by Serum Anti Mülerrian Hormone. PloS One. 2016;11:p.e0156130.

8. Ashrafi M, Arabipoor A, Hemat M, et al. The impact of the localization of endometriosis lesions on ovarian reserve and assisted reproduction techniques outcomes. J Obstet Gynaecol. 2018;39:91-97.

9. Romanski P, Brady P, Farland L, et al. The effect of endometriosis on the antimüllerian hormone level in the infertile population.J Assist Reprod Genet. 2019;36:1179-1184.

10. Broer S, Broekmans F, Laven J, et al. Anti-Müllerian hormone: ovarian reserve testing and its potential clinical implications. Hum Reprod Update. 2014;20:688-701.

11. Ling M, Murali M. Analysis of the Complement System in the Clinical Immunology Laboratory. Clin Lab Med. 2019;39:579-590.

12. Costabile M. Measuring the $50 \%$ Haemolytic Complement $(\mathrm{CH}<-$ sub $>50</$ sub $>$ ) Activity of Serum. J Vis Exp. 2010;37:1-3.

13. Kasapoglu I, Ata B, Uyaniklar O, et al. Endometrioma-related reduction in ovarian reserve (ERROR): a prospective longitudinal study. FertilSteril. 2018;110:122-127. 